\title{
TITLE:
}

\section{Flight Simulation using a Brain-Computer Interface: A Pilot, Pilot Study}

\section{AUTHORS:}

Michael Kryger ${ }^{1}$, Brock Wester ${ }^{2}$, Eric A. Pohlmeyer ${ }^{2}$, Matthew Rich ${ }^{2}$, Brendan John ${ }^{2}$, James Beaty $^{2}$, Michael McLoughlin ${ }^{2}$, Michael Boninger ${ }^{1,3-5,6}$, Elizabeth C. Tyler-Kabara ${ }^{1,5-7}$ 


\section{Abstract}

As Brain-Computer Interface (BCl) systems advance for uses such as robotic arm control it is postulated that the control paradigms could apply to other scenarios, such as control of video games, wheelchair movement or even flight. The purpose of this pilot study was to determine whether our $\mathrm{BCl}$ system, which involves decoding the signals of two 96-microelectrode arrays implanted into the motor cortex of a subject, could also be used to control an aircraft in a flight simulator environment.

The study involved six sessions in which various parameters were modified in order to achieve the best flight control, including plane type, view, control paradigm, gains, and limits. Successful flight was determined qualitatively by evaluating the subject's ability to perform requested maneuvers, maintain flight paths, and avoid control losses such as dives, spins and crashes.

By the end of the study, it was found that the subject could successfully control an aircraft. The subject could use both the jet and propeller plane with different views, adopting an intuitive control paradigm. From the subject's perspective, this was one of the most exciting and entertaining experiment she had done in two years of research.

In conclusion, this study provides a proof-of-concept that traditional motor cortex signals combined with a decoding paradigm can be used to control systems besides a robotic arm for which the decoder was developed. Aside from possible functional benefits, it also shows the potential for a new recreational activity for individuals with disabilities who are able to master $\mathrm{BCl}$ control.

\section{Keywords}

Brain-Computer Interface

Flight

\section{Abbreviations}

$\mathrm{BCl}$ - Brain-Computer Interface

MPL- Modular Prosthetic Limb

DARPA- Defense Advanced Research Projects Agency 


\section{Introduction}

The study of Brain-Computer Interfaces (BCls) is expanding at a rapid pace (Brunner et al., 2015). This is, in part, made possible by various advancements in hardware and software, including data acquisition using neural interfaces and imaging modalities (Utah Array (Maynard et al., 1997)), sheer computing power to process signals in real-time, and improved functionality of rehabilitative technologies. $\mathrm{BCls}$ have enabled users to interact with their environment, including exoskeletons (Kazerooni et al., 2005), anthropomorphic robotic arms such as the Johns Hopkins University Applied Physics Laboratory Modular Prosthetic Limb (MPL) (Johannes MS, 2011), and computer software used for communication, leisure, environmental control, and work productivity (Ebrahimi et al., 2003; Karmali et al., 2000; Krepki et al., 2007; Moore, 2003; Wolpaw et al., 2002).

Several surveys have explored the desired functional aspects of $\mathrm{BCls}$ in patients with spinal cord injuries and motor neuron disease (Anderson, 2004; Blabe et al., 2015; Collinger et al., 2013a; Lahr et al., 2015). In a 2015 study, respondents rated their top four functions as: emergency communication, personal computer operation, robotic arms for self-feeding, and use of a power wheelchair for mobility (Huggins et al., 2015). In a survey conducted by our team, respondents with tetraplegia indicated that while bladder/bowel function was the most important aspect for restoring quality of life, arm/hand function, walking, and upper body/trunk strength were also very important goals (Collinger et al., 2013a). Huggins also asked similar questions of patients with Amyotrophic Lateral Sclerosis in 2011, who reported robotic arm use and motorized wheelchair control as important goals. Computer control was also desirable, but not as important. One case study of a 78 year old subject in a locked-in state found that a $\mathrm{BCl}$ system connected to a paint program provided a high degree of satisfaction and increased selfesteem. This subject ended up displaying her work at an art show (Holz et al., 2015). These surveys suggest that subjects seek to return to functional independence, especially when it involves use of an arm, mobility, and use of a computer.

$\mathrm{BCl}$ research has tended to focus on goals similar to those highlighted in these prior surveys, including robotic arm control, mobility devices such as exoskeletons, and computer use, both for games, communication and business (Ebrahimi et al., 2003; Krepki et al., 2007; Wolpaw et al., 2002). The authors' collaborative team, under the DARPA Revolutionizing Prosthetics Program (McLoughlin, 2009), implanted two intracortical electrodes in motor cortex of a subject. Cortical signs were used to progressively increase the complexity of control of the MPL. The subject ultimately achieved 10dimensional control of the MPL to perform reaching and grasping movements (Collinger et al., 2014; Collinger et al., 2013b; Wodlinger et al., 2015).

Success in achieving independent real-time control opens the possibility that a $\mathrm{BCl}$ could allow an individual with paralysis to participate in other activities that require computer control. The goal of this study was to explore the ability of a participant to use a $\mathrm{BCl}$ to control a non-anthropomorphic device by piloting aircrafts using a flight simulator. This is primarily a proof-of-concept demonstration, starting with $\mathrm{BCl}$ control of only a few basic aspects of aircraft control. 


\section{Methods \\ Study Participant}

The study participant was a 53 year old woman diagnosed with a variant of spinocerebellar degeneration, without cerebellar involvement, resulting in complete quadriplegia (Boninger et al.). She was implanted with two 96-microelectrode arrays (Blackrock Microsystems, Salt Lake City, UT) in her left motor cortex (Collinger et al., 2013b). The series of trials described here were performed approximately two years post-implantation, over fifteen weeks. The study was conducted under an InvestigationalDevice Exception with FDA approval for the microelectrode array. The subject provided informed consent prior to participation in all research associated with this project.

\section{Data Acquisition}

Neural signals were acquired from the intracortical microelectrode arrays using the NeuroPort Neural Signal Processor (Blackrock Microsystems, Salt Lake City, UT). Typically, the participant used her neural activity to control a prosthetic limb (MPL) (Collinger et al., 2014; Collinger et al., 2013b; Wodlinger et al., 2015). For these experiments, the existing MPL software and hardware communication interface control system (VulcanX) (Bishop et al., 2008; Ravitz et al., 2013) was utilized to transform velocity commands into control signals for the flight simulator. Neural firing rates were transformed into two dimensional velocity control signals for the MPL using a two-step calibration process as previously described (Collinger et al., 2013b). A neural decoder was built to control 2D endpoint velocity of the MPL. Typically, the participant controlled 2D translational velocity (up/down and left/right), although, we also tested rotational velocity (pitch and roll). During the first step of calibration, the participant watched the MPL move to targets in the appropriate workspace while she attempted to perform the movements herself. An initial neural decoder was built to relate neural firing rate to endpoint velocity. This decoder was used in a second step of calibration where the subject controlled the MPL using neural signals while the computer constrained the movements directly towards or away from the targets. A final neural decoder was built from this second calibration. This calibration was completed each testing day. While the relationships between imagined movement and directional control were used as the starting point of the aircraft control sessions, no effort was made to force the simulated aircraft to directly mimic limb motions. Instead, these mapping signals provided a starting point for the participant to learn how to work in 'plane space' and obtain effective direct control of the aircraft's control surfaces (Figure 1). As a result, the participant had to use neural commands to learn how to effectively manipulate the control surfaces to achieve the desired aircraft responses. As the neural data was processed through VulcanX, there were additional parameters that provided extra limits and transformations of the data. These included (1) a threshold filter that excluded signals near zero to reduce noise, and (2) a transformation of the neural signal into scaled fractions of full deflections to reduce the overall complexity of control, and allow for faster changes in commands.

The neurally-commanded control surfaces included the elevators, used to control aircraft pitch (up and down movements of the aircraft nose), and the ailerons, used to control aircraft roll (rotation about the nose-to-tail axis of the aircraft). Control of the surfaces did not map in a 1:1 fashion to joint and endpoint movements of the prosthetic limb, as prosthetic limb control is velocity based (Clanton, 2011; 
Georgopoulos et al., 1986). For example, the velocities of the up and down wrist pitch were mapped to elevator positions. However, a neural command that caused the hand to pitch would not result in a constant nose up velocity of the aircraft. Instead, it resulted in positive deflection of the elevator that in turn caused the nose of the aircraft to pitch up at a greater than linear rate. Similarly, the neural command to rotate the limb wrist (pronation/supination) was mapped to the aileron positions. However, a neural command that caused a constant rotation of the limb hand had various impacts depending on the design of the aircraft. In the case of a jet aircraft that was tested (F-35), a constant aileron displacement caused a barrel roll of various speeds. In the case of the simpler propeller-driven aircraft (Mooney Bravo) that was tested, a constant wrist velocity command resulted in a turn of steadily decreasing radius. For either aircraft, changes in the positions of the ailerons impacted speed, which in turn affected how any given control surface position impacted the aircraft handling (i.e. a single control surface position does not have a fixed impact on the plane, but rather a variable one depending on the current speed and thus the previous commands/states in both control surfaces). While the subject was able to control aircraft heading as described above, she did not have control of acceleration during this process; instead, the aircraft was assigned a constant thrust.

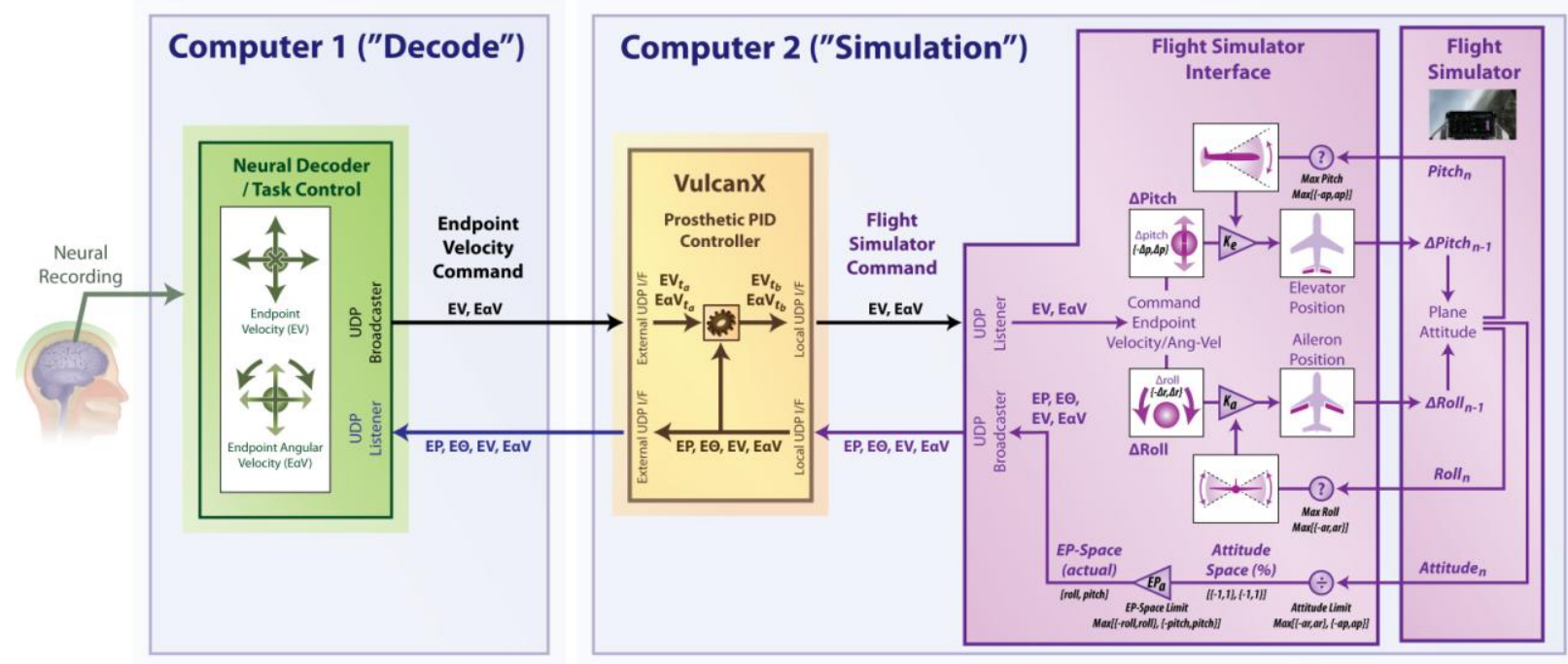

Figure 1- Flow of information to control an aircraft in a flight simulator. Existing software and hardware systems components were utilized to acquire, decode, and convert neural signals to control commands sent through the VulcanX controller and to the flight simulator. Endpoint velocity commands were transformed into deflection commands of the aircraft control surfaces." (UDP = User Datagram Protocol, EP = Endpoint Position, EO = Endpoint Orientation, ar = Attitude limits for roll , ap = Attitude Limits for pitch) 


\section{Study Sessions}

This study involved six different sessions with varying parameters used to assess the participant's ability to control a simulated aircraft in flight. Parameters that were modified included airplane type (which impacted how the aircraft body responded to movements of the control surfaces/neural inputs), point of view (a first person pilot view or an 'out of body' external chase view), gains and thresholds related to converting commands from arm space to plane space, changes in environment and visual feedback (aircraft location, altitude, and accompanying scenery), as well as

\begin{tabular}{|c|c|}
\hline Parameter & Description \\
\hline Airplane Type & $\begin{array}{c}\text { Mooney Bravo Propeller Plane } \\
\text { F-35 Fighter Jet }\end{array}$ \\
\hline Airplane View & $\begin{array}{c}\text { Cockpit } \\
\text { External Chase }\end{array}$ \\
\hline $\begin{array}{l}\text { Control } \\
\text { Paradigm }\end{array}$ & $\begin{array}{l}\text { Hand Rotation } \\
\text { Hand Translation }\end{array}$ \\
\hline Gains & $\begin{array}{l}\text { Roll/Pitch from Neural Controller } \\
\text { and Flight Sim }\end{array}$ \\
\hline Limits & Roll/Pitch- linear and nonlinear \\
\hline Location & $\begin{array}{c}\text { Grand Canyon, Pittsburgh, Cairo, Paris, } \\
\text { Honolulu, New York, St. Louis, Istanbul, } \\
\text { Las Vegas, Rio de Janeiro }\end{array}$ \\
\hline Task Type & $\begin{array}{c}\text { Free Flying } \\
\text { Hoop Challenge } \\
\text { Maneuver Time }\end{array}$ \\
\hline
\end{tabular}

Table 1- Parameters modified during test sessions various task types (Table 1 ).

\section{Data Analysis}

As an exploratory study, the data was evaluated qualitatively including data related to the participant's experience as described during the study sessions. The degree to which the study participant could control the aircraft was evaluated qualitatively based on how well she could perform requested maneuvers, maintain flight paths, and avoid control losses such as dives, spins and crashes. 


\section{Results}

\section{Overview}

The study participant was able to successfully pilot an aircraft through various courses using her decoded intracortical signals (Video 1 ). While there was some variability in ease of trials based on parameters of the decoder (e.g. noise suppression thresholds, deflection gains), in general, the participant was able to achieve good control immediately after training. The participant was able to achieve controlled changes in both pitch and roll fairly well, but it was often difficult for her to control each independently. Figure 2 shows one example of the subject's ability to navigate a specified course during the third session. During this session, the subject became capable of controlled descent to land the plane (although she did not control landing gear, brakes, etc.).
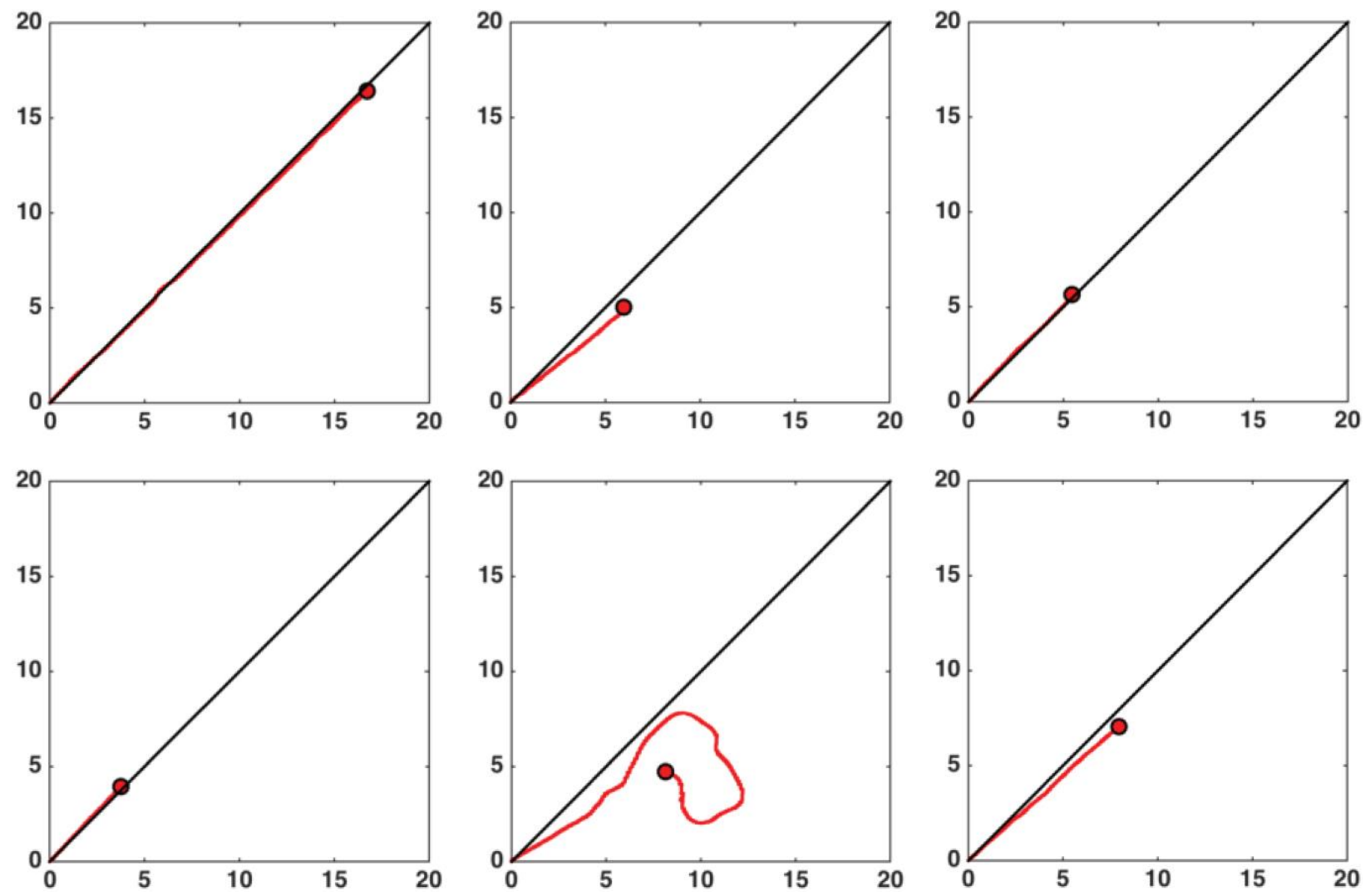

Figure 2- Sample flight paths to demonstrate subject's ability to stay on course during multiple trials. The black diagonal line is the instructed course. The red line with completion dot indicated ability to stay on course. Axes are all in kilometers. Note that only 1 trial significantly deviated from the intended course.

The participant reported that during the initial sessions, she would begin by visualizing hand movements to control the aircraft; however, within only a few trial runs, she quickly transitioned to simply visualizing the aircraft moving in the intended direction. Periodically, if the aircraft moved significantly in a direction the participant was not expecting, she would return to visualizing hand movements in an attempt to correct the course, but the participant described that her predominate strategy was to rely on visualizing desired aircraft movements. 


\section{Parameters}

Various observations were made regarding how to best optimize ease of flight, successfully carry out directions for aircraft control, and prevent course deviations. With regard to airplane type, the participant was able to control a propeller-driven plane (Mooney Bravo) better than a fighter jet (F-35). By the fifth study session, the participant subjectively improved control of the fighter jet as well. In general, during the initial session, the participant seemed to have an easier time controlling the plane when using an external chase view, rather than a cockpit view. However, after several sessions, the participant began to prefer the cockpit view for F-35 flight. This suggests that either view may be viable with practice, and a preferred view may change based on the experience of the user.

The participant tended to transition better from arm control to aircraft control when the parameters chosen to initialize the neurally-driven aircraft control surface commands were based on hand rotation movements rather than hand translations. The participant initially required limits on the degree of pitch and roll ( -20 to +30 degrees, and -20 to +20 degrees, respectively) that the aircraft was allowed to undergo to prevent control losses. However, after 2 sessions, the participant was able to control the aircraft without these limits. Once optimized values were determined for other parameters, such as gains, elevator and aileron deflections, these parameters resulted in consistent results over the six study sessions.

The participant was able to navigate through the Grand Canyon with surprisingly little difficulty (Video 1) despite the additional constraints of the canyon walls on each side. She accomplished this in the first session with less than one hour of piloting experience. Additionally, the participant felt the increased quantity and nature of the visual feedback from the canyon walls was valuable to improve her control skills as she attempted the run. Compared to other tests that used hoops to indicate more precisely defined courses, the participant's overall enthusiasm appeared much higher for tests that used more dynamic environments such as the Grand Canyon that gave a strong impression of flying movement, even if the goal of the task (e.g. navigate a single heading as straight as possible, with periodic turns) was similar.

\section{Hoop Challenge}

The hoop challenge required a stricter flight path. (Video 2) This test illustrated that controlling a physical system as distinct as an aircraft provides novel challenges. The first time this test was performed (with hoops indicating level flight at a single altitude), the participant showed gradual improvement, successfully navigating an increasing number of consecutive hoops. Initially, often only 13 hoops were navigated successfully, while in the following session, the participant was able to fly through 10 of 11 hoops by her third trial. It is likely that additional awareness of the aircraft's interaction within the flight simulator environment and impact of command decisions on subsequent aircraft control (e.g. avoiding the directing of the nose up to prevent stalling) played a role in improved performance. 


\section{Maneuver Time}

In other evaluations, the participant was instructed to roll the aircraft as quickly as possible in specific directions (clockwise and counterclockwise as visually indicated by a desired roll angle). The participant was able to perform these rolls on command in the specified directions quite well (Figure 3). Maneuver time was used instead of reaction time as the time taken involves not only the time for the participant to react, but also for the plane to begin to move.
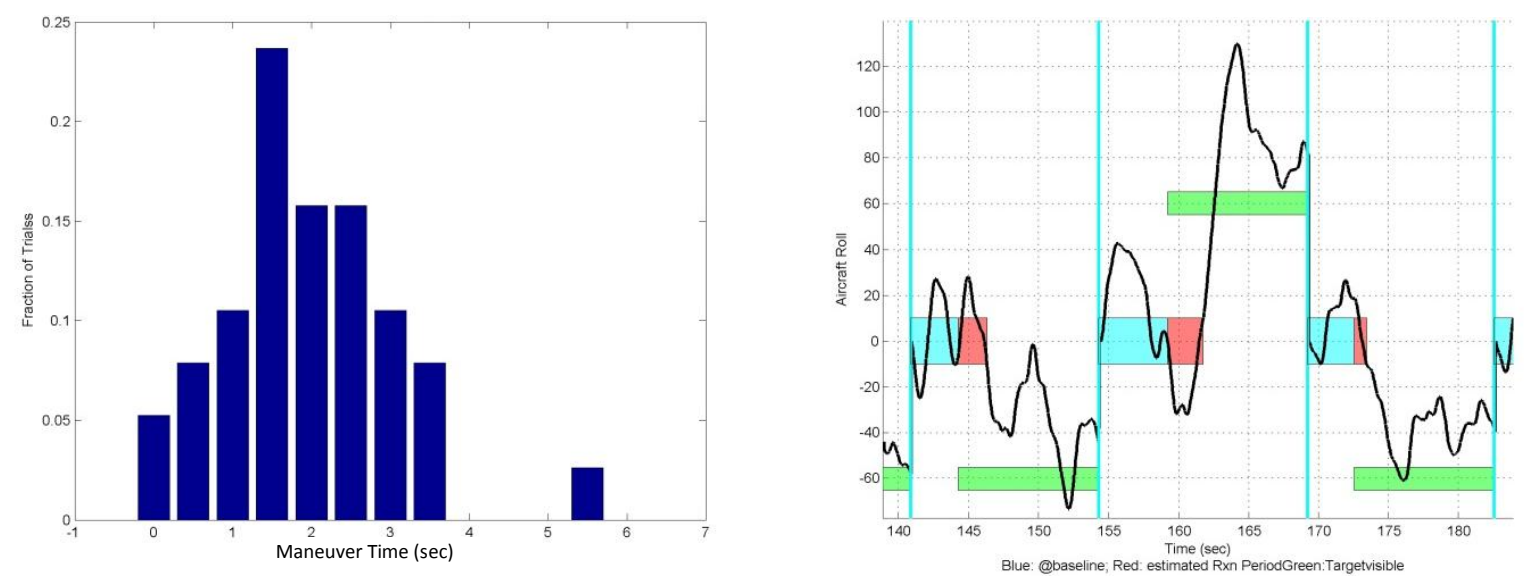

Figure 3- Maneuver Times. Left panel is the distribution of maneuver times when rolling to either the $+/$-10degree target (F35). Right panel shows three example trials. Blue boxes indicate inter-trial periods when the control signal (and aircraft) were around the neutral position, green boxes indicate the appearance and value of the instructed roll goals, and red boxes indicate the elapsed time to roll 10 degrees towards the target roll (used to estimate the maneuver time). Experiment was performed using an F-35. Note that the zero-second maneuver times signify instances where the aircraft was already in the process of rolling when the time started.

\section{Participant Impression}

From the perspective of the study participant, this set of experiments was a very positive experience. After two years of $\mathrm{BCl}$ experiments directed toward control of an advanced prosthetic, the participant reported that "this might be the coolest day since the first day", and that she was "flying in more ways than one". She was so immersed in the experience that she felt like she "left her body behind." She even made requests to fly to certain areas, such as Rio de Janeiro to see the "Christ the Redeemer" statue. In general, the participant found the control experience of the plane very "instinctive" using several strategies, including focusing on target destination, the movements of the airplane body, or (during initial sessions) envisioned hand movements. 


\section{Discussion}

Based on a literature review*, this study marks the first use of a flight simulator controlled by intracortical signals. A previous study in 2000 described using visual evoked potential EEG recordings to control aircraft roll only (Middendorf et al., 2000). A study in 2011 also used EEG to control a virtual helicopter (Doud et al., 2011). In 2014, a study demonstrated control of a physical quadcopter using EEG signals and eye tracking (Kim et al., 2014). Both of these rotorcraft-based systems require a somewhat different control paradigm with simpler mapping of directions. The control achieved with this study's system included the fundamental control methods of an airplane (the elevators and ailerons) allowing a large range of free flight movements, with tests involving flying through hoop courses and narrow canyons, and evaluations of take-offs and landings in a variety of engaging environments.

This study demonstrates the potential for generalizing $\mathrm{BCl}$ control using intracortical electrodes to systems other than robotic arm control. While restoration of arm function is a high priority for people with tetraplegia (Anderson, 2004; Blabe et al., 2015; Collinger et al., 2013a; Huggins et al., 2015; Lahr et al., 2015) and is rightly a primary focus of current $\mathrm{BCl}$ efforts, this research demonstrates that there may be other avenues in which to leverage $\mathrm{BCls}$. At the least, this proved to be a very enjoyable experience for the participant. It allowed her to experience travel that would otherwise be extremely difficult, enabling her to visit multiple countries without leaving the room. This is consistent with prior literature that has evaluated the importance of leisure activity in patients' lives (Holz et al., 2015).

In addition to leisure, this may also have practical applications in the future, such as allowing people with tetraplegia to control larger vehicles such as wheelchairs and cars. As this study has shown, the brain has the potential to use $\mathrm{BCl}$ s that are originally designed to control an anthropomorphic system (i.e. a robotic limb) to control physical systems that have different physical requirements, whether by visualizing a hand moving by itself, or via instinctively controlling a plane; the participant successfully achieved both types of control, quickly transitioning from one to the other. Notably, in none of the sessions did the participant report any feelings of nausea or motion sickness, or any similar complaint that can occur in virtual environments presenting inertial motion (Stoffregen et al., 2000). Despite making many different movements in the environment (rolls, dives, etc.) that could have resulted in feeling out of control, the participant remained very engaged. Even during periods in which she reported control errors or limits, she never reported being discomforted by the experience and instead repeatedly reported high levels of enjoyment.

In order to fully evaluate a $\mathrm{BCl}$-controlled flight system, further work should focus on quantifying successful control of a dynamic system, such as consistently achieving flights using hoop systems or engaging (but still quantifiable) environments to make sure the participant can follow a course. Such an evaluation could also use such metrics as reaction time as measured by the neural signal, and take into account the 'physical' restriction and response times of the system being controlled (i.e. given a command, how quickly the aircraft is capable of responding). It is also important to evaluate situations in which the aircraft might deviate from a desired course and require corrective action by the user. For example, occasionally a command to move up resulted in stalls and a counterintuitive loss of altitude. 
This could be considered part of learning to fly, and thus might be different in those who are familiar with conventional flight control principles.

\section{Conclusion}

This study demonstrates that an individual can successfully control an aircraft via a flight simulator using intracortical signals. This finding shows the potential of the brain to adapt to varying control paradigms, and could potentially lead to people with tetraplegia operating vehicles on their own in the future, beyond wheelchairs (Khare et al., 2011; Leeb et al., 2007). Importantly, this demonstrates a very gratifying simulation for $\mathrm{BCl}$ users to experience other cities and travel without leaving the ground. It provides an exposure to recreational activities which can help motivate training and provide new opportunities to study how the brain can interact with the environment beyond the constraints of the body. Future efforts should be focused on quantifying success rates for piloting, and optimizing parameters in order to make this a viable endeavor.

* Literature review for prior work related to flight control and brain computer interfaces was conducted by searching Pubmed for "flight" and Mesh term "Brain-Computer Interface", and Google Scholar search for "Brain-Computer Interface" and "flight".

\section{Acknowledgements}

This study is funded by the Defense Advanced Research Projects Agency's (Arlington, VA, USA) Revolutionizing Prosthetics program (contract number N66001-10-C-4056), Office of Research and Development, Rehabilitation Research and Development Service, Department of Veterans Affairs (Washington DC, USA, grant numbers B6789C, B7143R, and RX720), and UPMC Rehabilitation Institute (Pittsburgh, PA,USA). This study was done under an investigational device exemption granted by the US Food and Drug Administration. We would like to thank Jennifer Collinger for all of her support setting up these experiments, as well as her input during editing. We once again thank Jan Scheuermann for her extraordinary commitment and effort in relation to this study and insightful discussions with the study team; Karina Palko for her participation as an honorary research team member and support of the study; Elke Brown (Physical Medicine and Rehabilitation) for assistance with $\mathrm{BCl}$ testing sessions; Debbie Harrington (Physical Medicine and Rehabilitation) for regulatory management of the study; the University of Pittsburgh Clinical and Translational Science Institute and the Office of InvestigatorSponsored Investigational New Drugs and Investigational Device Exemption support for assistance with protocol development and regulatory reporting and compliance; the volunteer members of the DSMB for their continued monitoring of this study; Blackrock Microsystems (Salt Lake City, UT, USA) for coordination efforts and technical support in relation to this project. The views expressed herein are those of the authors and do not represent the official policy or position of the Department of Veterans Affairs, Department of Defense, or US Government. 


\section{References}

Anderson, K.D., 2004. Targeting recovery: priorities of the spinal cord-injured population. Journal of neurotrauma 21, 1371-1383.

Bishop, W., Armiger, R., Burck, J., Bridges, M., Hauschild, M., Englehart, K., Scheme, E., Vogelstein, R.J., Beaty, J., Harshbarger, S., 2008. A real-time virtual integration environment for the design and development of neural prosthetic systems, Engineering in Medicine and Biology Society, 2008. EMBS 2008. 30th Annual International Conference of the IEEE. IEEE, pp. 615-619.

Blabe, C.H., Gilja, V., Chestek, C.A., Shenoy, K.V., Anderson, K.D., Henderson, J.M., 2015. Assessment of brain-machine interfaces from the perspective of people with paralysis. Journal of neural engineering $12,043002$.

Boninger, M., Mitchell, G., Tyler-Kabara, E., Collinger, J., Schwartz, A.B., Neuroprosthetic control and tetraplegia - Authors'reply. The Lancet 381, 1900-1901.

Brunner, C., Birbaumer, N., Blankertz, B., Guger, C., Kübler, A., Mattia, D., Millán, J.d.R., Miralles, F., Nijholt, A., Opisso, E., Ramsey, N., Salomon, P., Müller-Putz, G.R., 2015. BNCI Horizon 2020: towards a roadmap for the $\mathrm{BCl}$ community. Brain-Computer Interfaces 2, 1-10.

Clanton, S., 2011. Brain-computer interface control of an anthropomorphic robotic arm, Robotics Institute. Carnegie Mellon University, Pittsburgh, Pennsylvania.

Collinger, J.L., Boninger, M.L., Bruns, T.M., Curley, K., Wang, W., Weber, D.J., 2013a. Functional priorities, assistive technology, and brain-computer interfaces after spinal cord injury. Journal of rehabilitation research and development 50, 145.

Collinger, J.L., Kryger, M.A., Barbara, R., Betler, T., Bowsher, K., Brown, E.H., Clanton, S.T., Degenhart, A.D., Foldes, S.T., Gaunt, R.A., 2014. Collaborative Approach in the Development of High-Performance Brain-Computer Interfaces for a Neuroprosthetic Arm: Translation from Animal Models to Human Control. Clinical and translational science 7, 52-59.

Collinger, J.L., Wodlinger, B., Downey, J.E., Wang, W., Tyler-Kabara, E.C., Weber, D.J., McMorland, A.J., Velliste, M., Boninger, M.L., Schwartz, A.B., 2013b. High-performance neuroprosthetic control by an individual with tetraplegia. The Lancet 381, 557-564.

Doud, A.J., Lucas, J.P., Pisansky, M.T., He, B., 2011. Continuous three-dimensional control of a virtual helicopter using a motor imagery based brain-computer interface. PloS one 6, e26322.

Ebrahimi, T., Vesin, J., Garcia, G., 2003. Brain-computer interface in multimedia communication. IEEE Signal Processing Magazine 20, 14-24.

Georgopoulos, A.P., Schwartz, A.B., Kettner, R.E., 1986. Neuronal population coding of movement direction. Science 233, 1416-1419.

Holz, E.M., Botrel, L., Kaufmann, T., Kübler, A., 2015. Long-term independent brain-computer interface home use improves quality of life of a patient in the locked-in state: a case study. Archives of physical medicine and rehabilitation 96, S16-S26.

Huggins, J.E., Moinuddin, A.A., Chiodo, A.E., Wren, P.A., 2015. What would brain-computer interface users want: opinions and priorities of potential users with spinal cord injury. Archives of physical medicine and rehabilitation 96, S38-S45. e35.

Johannes MS, B.J., Burck JM, Harshbarger SD, Kozlowski MV, Van Doren T, 2011. An overview of the developmental process for the modular prosthetic limb. Johns Hopkins APL Technical Digest 30, 207216.

Karmali, F., Polak, M., Kostov, A., 2000. Environmental control by a brain-computer interface, Engineering in Medicine and Biology Society, 2000. Proceedings of the 22nd Annual International Conference of the IEEE. IEEE, pp. 2990-2992. 
Kazerooni, H., Racine, J.-L., Huang, L., Steger, R., 2005. On the control of the berkeley lower extremity exoskeleton (BLEEX), Robotics and automation, 2005. ICRA 2005. Proceedings of the 2005 IEEE international conference on. IEEE, pp. 4353-4360.

Khare, V., Santhosh, J., Anand, S., Bhatia, M., 2011. Brain computer interface based real time control of wheelchair using electroencephalogram. International Journal of Soft Computing 1, 41-45.

Kim, B.H., Kim, M., Jo, S., 2014. Quadcopter flight control using a low-cost hybrid interface with EEGbased classification and eye tracking. Computers in biology and medicine 51, 82-92.

Krepki, R., Blankertz, B., Curio, G., Müller, K.-R., 2007. The Berlin Brain-Computer Interface (BBCI)towards a new communication channel for online control in gaming applications. Multimedia Tools and Applications 33, 73-90.

Lahr, J., Schwartz, C., Heimbach, B., Aertsen, A., Rickert, J., Ball, T., 2015. Invasive brain-machine interfaces: a survey of paralyzed patients' attitudes, knowledge and methods of information retrieval. Journal of neural engineering 12, 043001.

Leeb, R., Friedman, D., Müller-Putz, G.R., Scherer, R., Slater, M., Pfurtscheller, G., 2007. Self-paced (asynchronous) $\mathrm{BCl}$ control of a wheelchair in virtual environments: a case study with a tetraplegic. Computational intelligence and neuroscience 2007.

Maynard, E.M., Nordhausen, C.T., Normann, R.A., 1997. The Utah intracortical electrode array: a recording structure for potential brain-computer interfaces. Electroencephalography and clinical neurophysiology 102, 228-239.

McLoughlin, M.P., 2009. DARPA Revolutionizing Prosthetics 2009. DTIC Document.

Middendorf, M., McMillan, G., Calhoun, G., Jones, K.S., 2000. Brain-computer interfaces based on the steady-state visual-evoked response. IEEE Transactions on Rehabilitation Engineering 8, 211-214. Moore, M.M., 2003. Real-world applications for brain-computer interface technology. Neural Systems and Rehabilitation Engineering, IEEE Transactions on 11, 162-165.

Ravitz, A., McLoughlin, M., Beaty, J., Tenore, F., Johannes, M., Swetz, S., Helder, J., Katyal, K., Para, M., Fischer, K., Gion, T., BA, W., 2013. Revolutionizing prosthetics - phase 3. Johns Hopkins APL Technical Digest 31, 366-376.

Stoffregen, T.A., Hettinger, L.J., Haas, M.W., Roe, M.M., Smart, L.J., 2000. Postural instability and motion sickness in a fixed-base flight simulator. Human Factors: The Journal of the Human Factors and Ergonomics Society 42, 458-469.

Wodlinger, B., Downey, J., Tyler-Kabara, E., Schwartz, A., Boninger, M., Collinger, J., 2015. Tendimensional anthropomorphic arm control in a human brain- machine interface: difficulties, solutions, and limitations. Journal of neural engineering 12, 016011.

Wolpaw, J.R., Birbaumer, N., McFarland, D.J., Pfurtscheller, G., Vaughan, T.M., 2002. Brain-computer interfaces for communication and control. Clinical neurophysiology 113, 767-791. 